\title{
SHORT COMMUNICATION Size Effects in the Thermal Variations of the Hall Coefficient
}

\author{
C. R. PICHARD, C. R. TELLIER and A. J. TOSSER \\ Laboratoire d'Electronique, Université de Nancy-I, C.O. 140, 54037-Nancy Cedex France.
}

(Received August 17, 1979)

A number of experimental investigations of the temperature $T$ dependence of the Hall coefficient $R_{H F}$ of thin metallic films have been reported ${ }^{1-9}$ in the past few years. However some data have only been interpreted in terms of the temperature dependence of the electron density $\eta^{7,8,9}$, even if the transport properties of these metallic films agree $^{3,6,7,9,10}$ with the well known FuchsSondheimer theory. ${ }^{11}$

In a previous paper ${ }^{12}$ we have derived an expression for the correction in the temperature coefficient, $\beta_{R H}$ of the Hall coefficient $R_{H F}$ of thin film arising from the temperature dependence of the bulk mean free path $l_{0}$. Unfortunately in the case of partially specular scattering of electrons on the external surfaces the expression of $\beta_{R H}$ is somewhat complicated and its numerical evaluation requires the use of a digital computer.

In order to allow an easier calculation this letter proposes an alternative approach to derive an analytical expression for $\beta_{R H}$ : it is taken into account that for nearly specular scattering on external surfaces the surface scattering can be treated with good approximation by the Cottey method ${ }^{13}$ which states that the film resistivity $\rho_{F}$ and its temperature coefficient $\beta_{F}$ are respectively given by: ${ }^{13,14}$

$$
\begin{gathered}
\rho_{0} / \rho_{F}=F(\mu)=\frac{3}{2} \mu\left\{\mu-\frac{1}{2}+\left(1-\mu^{2}\right) \ln \left(1+\mu^{-1}\right)\right\} \\
\beta_{F} / \rho_{0}=1-G(\mu) \cdot[F(\mu)]^{-1}
\end{gathered}
$$

where: ${ }^{14}$

$G(\mu)=\mu \frac{\mathrm{d} F(\mu)}{\mathrm{d} \mu}=\frac{3}{2} \mu\left\{3 \mu-\frac{3}{2}+\left(1-3 \mu^{2}\right) \ln \left(1+\mu^{-1}\right)\right\}$

Here $\beta_{0}$ is the temperature coefficient (t.c.r.) of the bulk resistivity $\rho_{0}$ which can be written in the form: ${ }^{15}$

$$
\beta_{0}=\mathrm{d} \ln \rho_{0} / \mathrm{d} T=-\mathrm{d} \ln l_{0} / \mathrm{d} T
$$

The physical parameter $\mu$ is related to the specularity parameter $p$ and to the reduced thickness $k$ by: ${ }^{13}$

$$
\mu=k \cdot\left[\ln \frac{1}{p}\right]^{-1} \approx k \cdot[1-p]^{-1} ; p \geqslant 0.5
$$

with: $k=a / l_{0}$.

We have previously shown ${ }^{16}$ that in the case of transverse magnetic field the Hall coefficient of thin metallic $R_{H F}$ can be expressed in terms of film resistivity $\rho_{F}$ and its t.c.r. $\beta_{F}$ :

$$
R_{H F} / R_{H O} \approx\left(\rho_{F} \cdot \beta_{F}\right) /\left(\rho_{0} \cdot \beta_{0}\right)
$$

where $R_{H O}$ is the Hall coefficient in bulk material.

By combining Eqs. (1), (2) and (6) we get:

$$
R_{H F} / R_{H O}=[1-D(\mu)] \cdot[F(\mu)]^{-1}
$$

with: $D(\mu)=G(\mu) / F(\mu)$

Taking into account the value of the bulk Hall coefficient $R_{H O}:^{7,17}$

$$
R_{H O}=-\frac{1}{\eta e}
$$

we get:

$$
\frac{\mathrm{d} R_{H} O}{R_{H O}}=-\frac{\mathrm{d} \eta}{\eta}
$$

Moreover Eq. (5) yields:

$$
\frac{\mathrm{d} \mu}{\mu}=-\frac{\mathrm{d} l_{0}}{l_{0}}
$$

assuming that thermal variations in thickness $a$ and specularity parameter can be neglected; the validity of these assumptions has been recently established. ${ }^{18}$

Logarithmic differentiation of Eq. (7) leads to: 


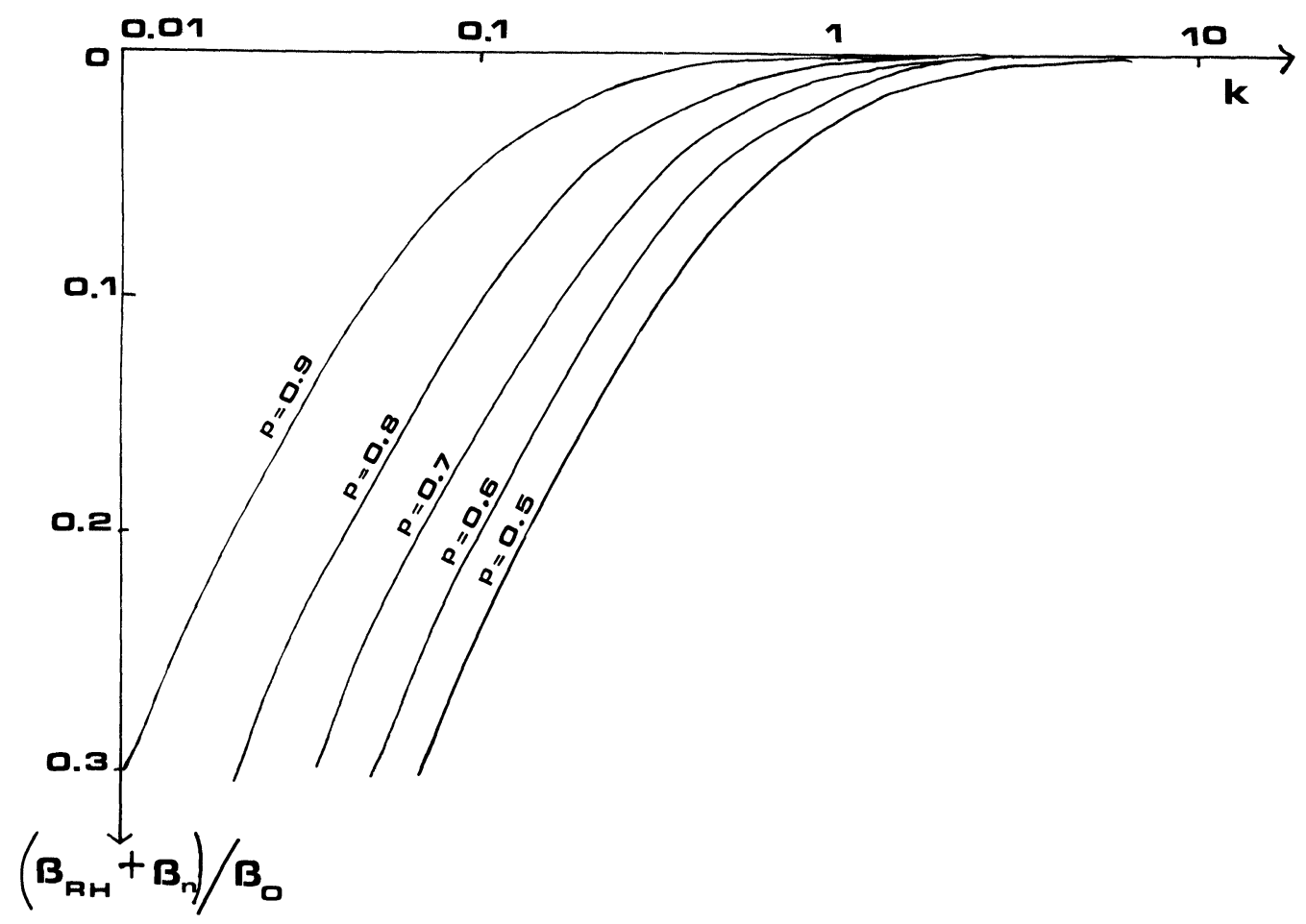

FIGURE 1 Theoretical plots of the ratio $\left(\beta_{R H}+\beta_{\eta}\right) / \beta_{0}$ versus the reduced thickness $k$ for some values of the specularity parameter $p$.

$$
\begin{aligned}
& \frac{\mathrm{d} R_{H F}}{R_{H F}}=-\frac{\mathrm{d} \eta}{\eta}+\frac{\mathrm{d} l_{0}}{l_{0}} \frac{D(\mu)}{1-D(\mu)}\left\{\frac{\mu}{G(\mu)} \frac{\mathrm{d} G(\mu)}{\mathrm{d} \mu}\right. \\
& \left.-\frac{\mu}{F(\mu)} \frac{\mathrm{d} F(\mu)}{\mathrm{d} \mu}\right\}+\frac{\mathrm{d} l_{0}}{l_{0}}\left\{\frac{\mu}{F(\mu)} \cdot \frac{\mathrm{d} F(\mu)}{\mathrm{d} \mu}\right\}
\end{aligned}
$$

For thin films which exhibit linear $R_{H F}$ versus $T$ plots as previously reported by some authors ${ }^{7}$ we may define the temperature coefficient $\beta_{R H}$ of Hall coefficients as:

$$
\beta_{R H}=\mathrm{d} \ln R_{H F} / \mathrm{d} T
$$

by combining Eqs. (3), (4) and (11) and rearranging we finally obtain:

$$
\beta_{R H}=-\beta_{\eta}-\beta_{0} \cdot D(\mu)\left\{\frac{C(\mu)-D(\mu)}{1-D(\mu)}+1\right\}
$$

where:

$$
C(\mu)=\frac{\mu}{G(\mu)} \cdot \frac{\mathrm{d} G(\mu)}{\mathrm{d} \mu}
$$

and:

$$
\begin{aligned}
\mu \frac{\mathrm{d} G(\mu)}{\mathrm{d} \mu}= & \frac{3}{2} \mu\left\{G(\mu)-\frac{3}{2}+\left(1-9 \mu^{2}\right) \ln \left(1+\mu^{-1}\right)\right. \\
& \left.-\frac{1-3 \mu^{2}}{1+\mu}\right\}
\end{aligned}
$$

$\beta_{\eta}$ is the temperature coefficient of the electronic density $\eta$ previously defined as: ${ }^{12}$

$$
\beta_{\eta}=\mathrm{d} \ln \eta / \mathrm{d} T
$$

Eq. (13) predicts that $\beta_{R H}$ depends on the reduced thickness $k$; this behaviour can be easily explained since it has been found that $R_{H F}$ markedly depends on film thickness ${ }^{15,17}$ for relatively thin films $(k<0.5)$.

Figure 1 shows theoretical plots of the ratio $\left(\beta_{R H}+\beta_{\eta}\right) / \beta_{0}$ for different values of the specularity parameter $p$. It is clear from Eq. (13) that $\beta_{R H}+\beta_{\eta}$ gives the expression of the change in temperature of the Hall coefficient due to the temperature dependence of the reduced thickness; hence, as 
expected, the ratio $\left|\left(\beta_{R H}+\beta_{\eta}\right) / \beta_{0}\right|$ decreases (for a given thickness) with increasing values of the specularity parameter $p$; moreover it appears that the magnitude of $\left(\beta_{R H}+\beta_{\eta}\right) / \beta_{0}$ rapidly decreases with increasing values of $k$ and finally vanishes for thick films (for example when $p=0.75$ for $k>0.1$ ).

More generally marked size effects could be observed only if the magnitude of $\beta_{\eta}$ is considerably smaller than the generally positive $\beta_{0}$ value. For example for $p=0.5$ and $k=0.5$ we obtain a deviation less than $20 \%$ until the ratio $\left|\beta_{\eta} / \beta_{0}\right|$ keeps values greater than 0.2 .

As a consequence of this study we estimate, as suggested in a previous paper, ${ }^{12}$ that the temperature dependence of the Hall coefficient of thin metallic films may be (in the relatively high temperature range $(T>150 \mathrm{~K}))$ interpreted in terms of thermal variations in both the electronic density $\eta(T)$ and the mean free path $\rho_{0}(T)$; nevertheless, this last effect is significant only if the magnitude of $\beta_{\eta}$ is considerably less than $\beta_{0}$.

Further insight is thus given for the interpretation of thermal variations in Hall coefficient of thin metallic films.

\section{REFERENCES}

1. R. A. Hoffman and D. R. Frank1, Phys. Rev. B, 3, (1971) 1825-1833.
2. N. Garcia, Y. H. Kao and M. Strongin, Phys. Rev. B., 5 (1972) 2029-2039.

3. A. Kinbara and K. Ueki, Thin Solid Films, 12 (1972) 63-66.

4. D. Golmayo and J. L. Sacedon, Thin Solid Films, 35 (1976) 143-147.

5. K. L. Chopra, R. Suri and A. P. Thakoor, J. Appl. Phys., 48 (1977) 538-546.

6. S. Chaudhuri and A. K. Pal, J. Appl. Phys., 48 (1977) $3455-3461$

7. M. Viard, J. P. Drexler and J. Flechon, Thin Solid Films, 35 (1976) 247-253.

8. L. S. Hsu, Y. Y. Chang, C. S. Young and P. K. Tseng, J. Appl. Phys., 47 (1976) 2359-2363.

9. M. Inoue, Y. Tamaki and H. Yagi, J. Appl. Phys., 45 (1974) 1562-1566.

10. J. Flechon, J. P. Drexler and M. Viard, Thin Solid Films, 31 (1976) 353-357.

11. E. H. Sondheimer, Adv. Phys., 1 (1952) 1-50.

12. C. R. Tellier, C. R. Pichard and A. J. Tosser, Electrocomp. Sci \& Technol. 6 (1979) 19-22.

13. A. A. Cottey, Thin Solid Films, 1 (1967/68) 297-307.

14. C. R. Tellier and A. J. Tosser, Thin Solid Films, 52 (1978) 53-61.

15. See for example, K. L. Chopra, Thin Film Phenomena. (McGraw Hill, 1969).

16. C. R. Tellier and A. J. Tosser, Rev. Phys. Appl., 13 (1978) 441-447.

17. C. R. Tellier, M. Rabel and A. J. Tosser, J. Phys. F: Metal Physics, 8 (1978) 2357-2365.

18. C. R. Tellier and A. J. Tosser, Thin Solid Films, 60 (1979) 91-95. 

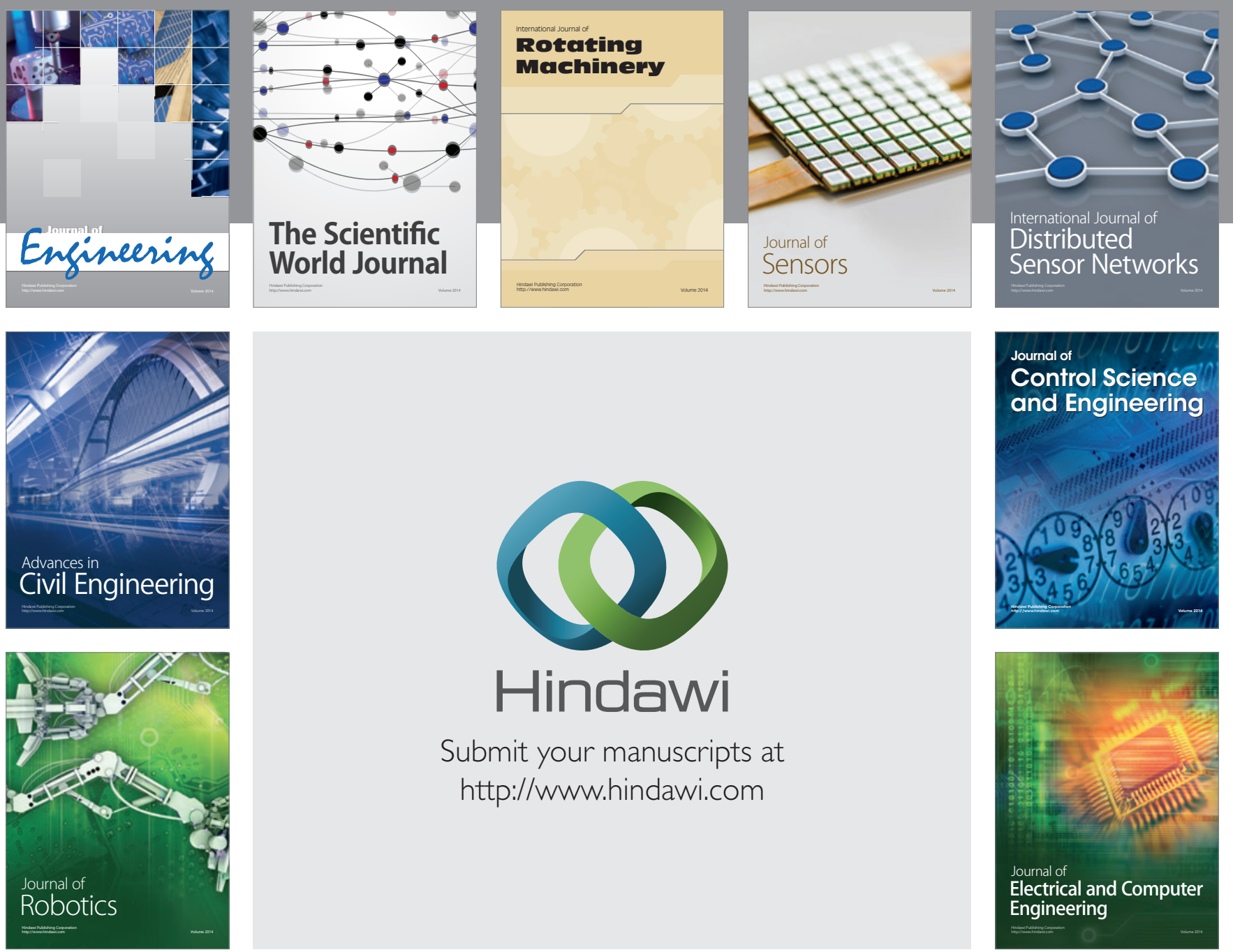

Submit your manuscripts at

http://www.hindawi.com
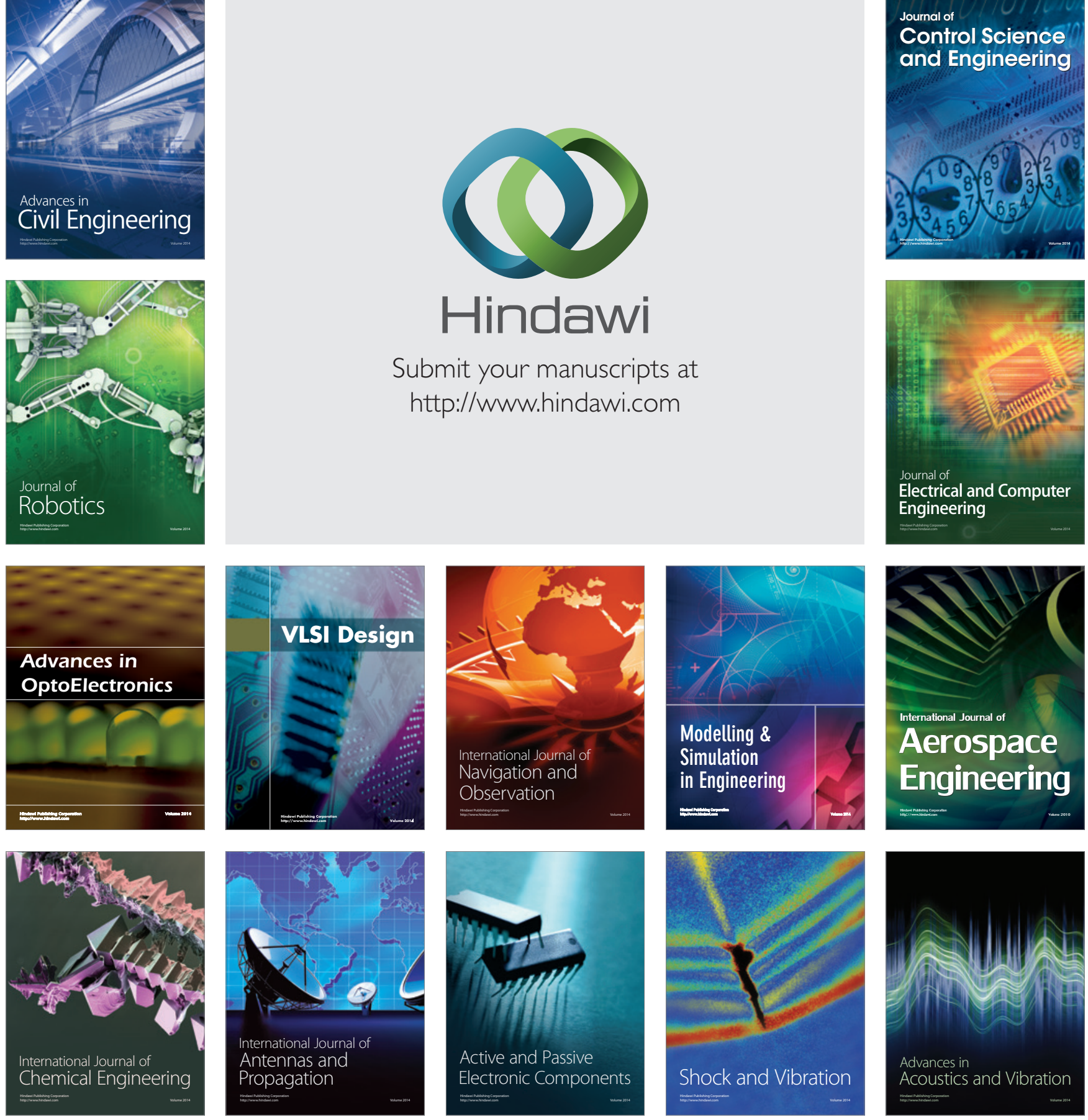